\title{
Synthesis methods of gold nanoparticles for Localized Surface Plasmon Resonance (LSPR) sensor applications
}

\author{
Nurul Diyanah Samsuri, Wan Maisarah Mukhtar ${ }^{*}$, Affa Rozana Abdul Rashid, Karsono Ahmad Dasuki, and Awangku \\ Abdul Rahman Hj. Awangku Yussuf
}

Faculty of Science and Technology, Universiti Sains Islam Malaysia, 71800 Malaysia

\begin{abstract}
Gold nanoparticles (GNPs) have been known as an excellent characteristic for Local Surface Plasmon Resonance (LSPR) sensors due to their sensitive spectral response to the local environment of the nanoparticle surface and ease of monitoring the light signal due to their strong scattering or absorption. Prior the technologies, GNPs based LSPR has been commercialized and have become a central tool for characterizing and quantifying in various field. In this review, we presented a brief introduction on the history of surface plasmon, the theory behind the surface plasmon resonance (SPR) and the principles of LSPR. We also reported on the synthetization as well of the properties of the GNPs and the applications in current LSPR sensors.
\end{abstract}

\section{Introduction}

Surface plasmon resonance (SPR) is a charge-density oscillation that may exist at the interface of two media with dielectric constants of opposite signs, for instance, a metal and a dielectric. The charge density wave is associated with an electromagnetic wave, the field vectors of which reach their maxima at the interface and decay evanescently into both media. This surface plasma wave (SPW) is a TM-polarized wave (magnetic vector is perpendicular to the direction of propagation of the SPW and parallel to the plane of interface) [1]. Due to the multidisciplinary nature of SPR, major areas of applications of SPR has been reported such as for measurement of physical quantities (displacement [2] and angular position [3]), chemical sensing (monitoring distillation processes [4]), and biosensing (first demonstrated in 1983 [5]).

Historically, in 1902, an experiment conducted by Wood which he had illuminated a metallic diffraction grating with polychromatic light was the first documented observation of surface plasmon. In 1958, an increased drop in reflectivity was observed by Thurbadar [6] as a thin metal film was being illuminated on a substrate without related this observation to SPR phenomena. In 1968, Otto described Thurbadar's observation by showing the excitation of surface plasmon resulted the drop in the reflectivity in the attenuated total reflection method [7]. This explanation also being supported by Kretschmann and Raether, which explained the same excitation of surface plasmon in the same year but with different configurations [8]. Thus, these observations of Otto, Kretschmann and Raether had entrenched and introduced the surface plasmon into modern optics [9]. The advantages of surface plasmon started being known in early 1980s when the method was used for detecting gases [10]. Ever since, various SPR sensors have been investigated.

In recent years, sensors based localized surface plasmon resonance (LSPR) had drawn more interest [11]. LSPR is also a SPR phenomenon but instead of existed in thin film metal, they are in the metallic nanoparticles (MNPs) form [12]. The physical properties of LSPR is reported to be more sensitive than the traditional bulk metal thin film based SPR sensors [13] due to the nanoscale size of the particles [14]. These improvements had attracted more significant development of the high sensitivity LSPR sensors. Among the MNPs, gold nanoparticles (GNPs) have been demonstrated as a great characteristic for a LSPR sensor due to its solitary optical properties [15].

\section{Basic Theory}

Surface plasmon is the free electrons at the interface of two materials located at a metal surface where free conduction electrons are abundant. This condition obeys the metal dielectric interface by Maxwell's equations [16] which can mathematically be described by an electric field $\boldsymbol{E}$ :

$$
E=E_{0} e^{-k_{y_{2}} y} \exp \left(j \omega t-j k_{x} x\right)
$$

where $E_{0}$ is the amplitude of the electric field, $\omega$ is the angular frequency, $\boldsymbol{k}$ is the wavevector, $(\boldsymbol{x}, \boldsymbol{y})$ is the position vector and $j=\sqrt{-1}$.

\footnotetext{
* Corresponding author: wmaisarah@usim.edu.my
} 
The propagating electron that occurring at the interface between metal and dielectric experienced an evanescence wave. Evanescent waves happened when incident wave is reflected at an angle that greater than the critical angle thus attenuated total reflection (ATR) occurred as shown in Figure 1.

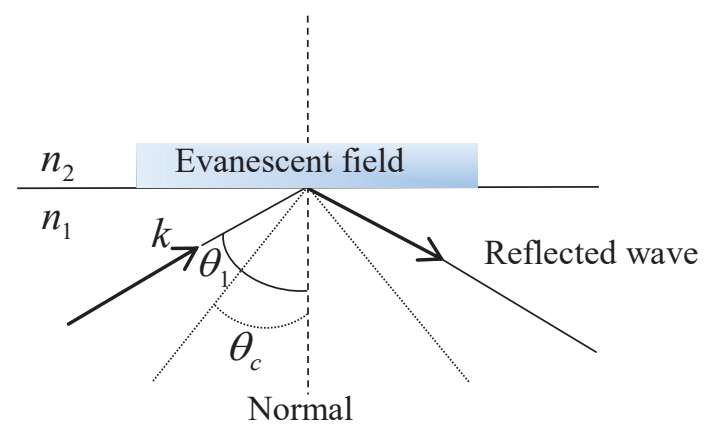

Fig. 1. Shown an interface consisting of two media with refractive indices $n_{2}<n_{1}$. An evanescent field generated when the angle of incidence is larger than the critical angle [17].

\section{Principles of LSPR}

LSPR is a phenomenon when the electromagnetic field of the light induces a collective coherent oscillation of the surface conduction electron in resonance with the frequency of light when incident light interacts with MNPs [18]. The excitation of LSPR occurred when a charge separation happened between the free electron and ionic metal core due to the interaction between the electric field of light and free electron in the nanoparticles. Thus, the restoring force (Coulomb repulsion among the free electron) forced the free electron to shift in different direction [19].

The phenomenon of LSPR shows a great absorption of light. Since each MNPs shows varies absorption properties hence the properties of LSPR is effected by the size, shape and the dielectric properties of the each MNPs [23]. As LSPR is exceptionally susceptible to the refractive index change in the local dielectric medium, the alteration of the peak wavelength or peak absorbance in the absorbance spectrum of MNPs in sensor system is exerted as a measure of the LSPR sensor feedback [35].

The Mie solution to Maxwell's equations can be applied to defined the light absorption of gold nanosphericals (GNSs) affected by LSPR [38]. Based on the Mie theory, the extinction cross-section, $C_{\text {ext }}$ for separated spherical nanoparticles with a radius $R$ (that is smaller than the wavelength of light $\lambda(R / \lambda<0.1))$, can be indicated as:

$$
C_{e x t}=\frac{24 \pi^{2} R^{3} N \varepsilon_{m}^{\frac{2}{3}}}{\lambda} \frac{\varepsilon_{i}}{\left(\varepsilon_{r}+2 \varepsilon_{m}\right)^{2}+\varepsilon_{i}^{2}}
$$

where $\varepsilon_{m}$ is the dielectric constant of the surrounding medium, $\varepsilon_{r}$ is the real part of the dielectric function of the MNPs, $\varepsilon_{i}$ is the imaginary part of the dielectric function of the MNPs and N is the number of spheres per unit volume.

According to Equation (2), the plasmon absorption band appears when $\varepsilon_{r}=-2 \varepsilon_{m}$. Figure 2 shows the excitation of LSPR for the GNSs.

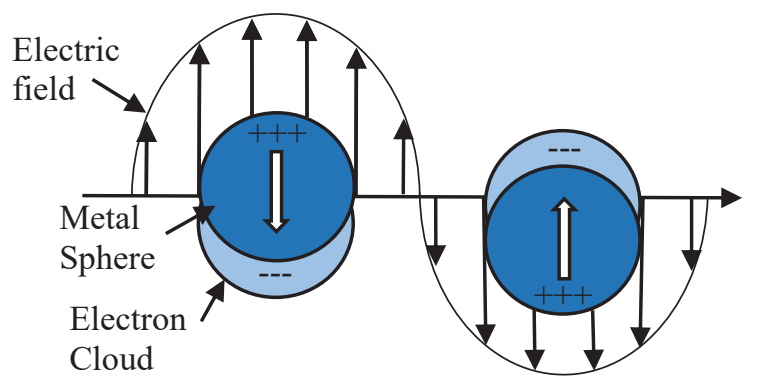

Fig. 2. Excitation Schematic illustration of LSPR excitation for GNSs [22].

\section{Synthesis of Gold Nanoparticles}

In prior years, citrate reduction method is being used for yielding GNPs by adding the GNPs into the boiling gold salt $\left(\mathrm{HAuCl}_{4}\right)$ solution where the citrate solution is known, thus, allowing the size of the GNPs to be controlled by varies the ratio between the gold salt and citrate solution [23]. In this section, a brief review of several typical synthesis methods is discussed.

\subsection{Chemical Method}

The simplest method for synthesize a GNPs is through a chemical method. Usually, the synthesized monodisperse spherical GNPs is range between 10 to $20 \mathrm{~nm}$ in diameter [24]. The GNPs is produced in the presence of reducing agents such as amino acid, ascorbic acid or citrate [25], which then reacted with a small quantity of hot gold salt. The citrate ions which act as reducing and capping agents helps the formation of colloidal gold. To produce a larger particle, the amount of sodium citrates need to be decreased so that the remaining amount will not be sufficient in reducing the gold. As the quantity of sodium citrate decreased, the quantity of the citrate ions needed for stabilizing the particles in the solution decreased. Hence, the small particles will start to accumulate into bigger particles [26].

In early 1990s, Brust and Schiffrin contrive the Brust method which used in synthesizing GNPs in an organic solution which are not mixable with water such as toluene. Tetra-octylammonium bromide (TOAB) solution is being reduced with the gold salt in toluene together with sodium borohydride $\left(\mathrm{NaBH}_{4}\right)$ which act as both an anti-coagulant and reducing agent. The GNPs produced usually in 2 to $6 \mathrm{~nm}$ in diameter. Here, the 
TOAB and $\mathrm{NaBH}_{4}$ both also acted as the stabilizing agent and phase transfer catalyst. In 1994, the Burst method is chosen as the popular synthesize technique as it gives the control in both particle diameter and grain size distribution also functionalization of particle surface with alkanethiols which helps in stabilized the GNPs [27]. This resulted in the color change from orange to brown in the reaction [28]. Purification of GNPs stabilized with dodecanethiol from TOAB was recorded by Schriffin [29].

\subsection{Seed-mediated Growth Method}

Due to its simplicity of the experimental procedure [30], seed-mediated growth has been the most favored and extensively used among the reported GNPs synthesis method. Plus, this method enable researchers to produce a high number of nanoparticles [31], the access in controlling the size of the particle [32] and the ease in structural alterations [33]. Basically, seed-mediated growth involved two important steps. Firstly, by producing a seed particles (reduced in gold salts with strong reducing agent) which then added to a metal salt solution in presence of weak reducing agent. Plus, to quicken the growth and avoid further nucleation of the GNPs, a structure directing agent also been added to the solution. Lastly, by varies the concentration of the seeds, reducing and structure directing agents; the geometry of the GNPs can be modified [34].

\subsection{Biological Method}

In recent years, the advancement of 'green chemistry' technology in GNPs synthesis is widely been progressed to study the biological applications [35]. By using different types of microorganisms, various morphology and chemical composition of GNPs have been synthesized and been studied in several medical technology areas [36] due to the safe, non-toxic and ecofriendly procedures [37]. These microorganisms being categorized into two group which are intracellular and extracellular depends on the location of the GNPs are growth [38]. Besides, plant extracts also being studied and used to synthesize a uniform size and shape of GNPs [39].

\section{Properties of Gold Nanoparticles}

The quantum size effect in GNPs resulted in individual electron transition energy levels. An external ligands, magnetic fields and electrolyte ions can be used to modulate the quantized capacitance charging behaviour of GNPs. This gives likely applications in electronic devices and electrochemical labels [40]. As for the redox activity, GNPs promoted SPR phenomena [41]. This phenomenon happened when a collective oscillation of conduction electrons across the nanoparticles occurred due to the excitation of the resonant from the incoming photon [42].
The colours from the GNPs is resulted from the resonance condition which meet at visible wavelength [43]. Besides size, SPR also is affected by the solvent, ligand and temperature [42].

A spectral shift created by solvent refractive index changes that are parallel with Mie theory have been reported by Murray et al. [44]. These core changes are crucial in affecting the shifting in the surface plasmon band (SPB) energy to a lower energy with electron deficiency and higher energy with surplus electronic charges [45]. Electronic dephasing can also affect the SPR [46] and has been suggested rather electron-phonon, only electron-electron interactions are implicated [47].

Nevertheless, the phenomena of both procedure in the individual GNPs has been validated from the femtosecond light scattering by experimental data [48]. A significant red-shifting (from $\sim 520$ to $\sim 650 \mathrm{~nm}$ ) and the solution changing from red to blue colour because of the interparticle plasmon coupling are the results from the aggregation of nanoparticles since SPR frequency is responsive to the vicinity of other nanoparticles [49]. This occurrence made GNPs a great particle for colorimetric sensors.

A theoretical calculation of the extinction coefficient of GNPs have been recorded by El Sayed et al. [41]. From the UV-Vis/extinction spectra, the size and concentration of GNPs have been certified both theoretically and experimentally [50]. Also, various stabilizing ligands of the extinction coefficients of the GNPs have been reported [51].

\section{Applications in LSPR Sensors}

Analyte adsorption which caused the local dielectric charges increased to the wavelength shift in the LSPR spectrum is the basic principle of LSPR based sensors. The conduction of LSPR assays happened in both solution phase [52] and with surfaces filmed with nanoparticle monolayers [53]. When an interaction occurred between monoclonal antibodies with GNPs functionalized and analytes, the absorption maxima of LSPR was redshifted [54]. Plus, the number of ligands is proportional to the wavelength shift [54]. Nevertheless, most GNPs based SPR sensors have been made to be immobilized nanoparticles onto surface [55]. To enhance the sensitivity of SPR, GNPs have been introduced onto the sensing surface due to its metal film on the surface, high dielectric constants and the electromagnetic coupling between GNPs [56]. For instance, by immobilizing an MIP gel with implanted GNPs, the level of dopamine in nanomolar concentration can be detected by using a gold film-coated chip [57]. Plus, as supports for assisted GNPs in identifying analytes such as human serum albumin, numerous substrates such as sol-gel matrix, optical fibers, quartz and ITO glass have been utilized [58]. Lastly, in discovering insulin, an immobilized hydroxyl/thiol-functionalized fourth generation PAMAM dendrimer capsulated GNPs were attached onto maleimide terminated SAMs [59]. An enhance sensitivity with detection limit of $0.5 \mathrm{pM}$ and 
increased stability were resulted from the GNPsmodified dendrimers. By studying human serum samples with good correlation to standard methods by comparing normal and diabetic patients, the sensor was further certified [60]. An alteration happened due to an increased in surface absorption band to the visible colour change caused by the GNPs aggregation. Mirkin et al. had utilized the above principles by using an oligonucletide functionalized GNPs both in surface and dispersions to develop a calorimetric sensor for DNA hybridization assay [59]. Meanwhile, other reported SPR based sensor used the GNPs aggregation to detect proteins [61] and lectin [62].

\section{Conclusion}

Based on the review, as compared to the traditional SPR sensors, LSPR GNPs based have been determined to be more sensitive and sensible in sensors applications due to its enhance optical properties. Hence, many researches had shown interest in studying on the development and improvement of GNPs based LSPR sensors in enhancing its sensor ability.

The authors would like to acknowledge the support of Malaysian Ministry of Higher Education (MOHE) through Universiti Sains Islam Malaysia (USIM) under grant USIM/FRGS/FST/32/51514.

\section{References}

1. S. S. Yee, Surface plasmon resonance sensors: review, 54, 3-15 (1999)

2. G. Margheri, A. Mannoni, F. Quercioli, Proc. SPIE 2783, 211-220 (1996)

3. J.K. Schaller, R. Czepluch, C.G. Stojanoff, Proc. SPIE 3098, 476-486 (1997)

4. E.G. Ruiz, I. Garcez, C. Aldea, M.A. Lopez, J. Mateo, J. Alonso-Chamarro, S. Alegret, Sensors and Actuators A 37-38, 221-225 (1993)

5. B. Liedberg, C. Nylander, I. Lundstro"m, Sensors and Actuators 4 299-304 (1983)

6. T Turbadar, Proc Phys Soc 73, 40 (1959)

7. A Otto, Zeits Phys 216, 398 (1968)

8. E Kretschmann, H Raether, Z Naturforsch 2135-2136, 2135-2136 (1968)

9. H Raether, Springer Tracts Mod Phys 111, 1 (1988)

10. C Nylander, B Liedberg, $\mathrm{T}$ Lind, Sensor Actuator 3, 79 (1982)

11. M. Potara, A.M. Gabudean, S. Astilean, Journal of Materials Chemistry 21, 3625-3633 (2011)

12. A.D. McFarland, R.P. Van Duyne, Nano Letters 3,1057-1062 (2003)

13. A.V. Kabashin, P. Evans, S. Pastkovsky, W. Hendren, G.A. Wurtz, R. Atkinson,R. Pollard, V.A. Podolskiy, A.V. Zayats, Nature Materials $8,867-871$ (2009)
14. S. Eustis, M.A. El-Sayed, Chemical Society Reviews 35, 209-217 (2006)

15. X. Huang, S. Neretina, M.A. El-Sayed, Advanced Materials 21,4880-4910 (2009)

16. R. O. B. P. H. Kooyman, Physics of Surface Plasmon Resonance, 15-34.

17. C. Soc, M. Schnippering, S. R. T. Neil, R. Mackenzie, P. R. Unwin, Chem Soc Rev 207220 (2011)

18. J.N. Anker, W.P. Hall, O. Lyandres, N.C. Shah, J. Zhao, R.P. Van Duyne, Nature Materials 7, 442-453 (2008)

19. J. Cao, T. Sun, K. T. V. Grattan, Sensors and Actuators B : Chemical, 195, 332-351 (2014)

20. K.M. Mayer, S. Lee, H. Liao, B.C. Rostro, A. Fuentes, P.T. Scully, C.L. Nehl, J.H.Hafner, ACS Nano 2, 687-692 (2008)

21. K.M. Mayer, J.H. Hafner, Chemical Reviews 111, 3828-3857 (2011)

22. K.A. Willets, R.P. Van Duyne, Annual Review of Physical Chemistry, Annual Reviews, Palo Alto 58, 267-297 (2007)

23. V.V.R. Sai, T. Kundu, S. Mukherji, Biosensors \& Bioelectronics 24, 2804-2809 (2009)

24. N. Sharma, G. Bhatt, P. Kothiyal, IJPBR 3(2), 13-27 (2015)

25. X. Ji, X. Song, J. Li, Y. Bai, W. Yang, X. Peng, Journal of the American Chemical Society 129, 13939-13948 (2007)

26. R.K. Gangwar, V.A. Dhumale, D. Kumari, U.T. Nakate, S.W. Gosavi, R.B. Sharma, S.N. Kale, S. Datar, Materials Science and Engineering : C 32, 2659-2663 (2012)

27. M. Giersig, P. Mulvaney, The ACS journal of surfaces and colloids 9, 3408-3413 (1993)

28. M. Faraday, Philosophical Transactions of the Royal Society of London 147, 145-181 (1857)

29. C.A. Waters, A.J. Mills, K.A. Johnson, D.J. Schiffrin, Chemical Communications, 540-541 (2003)

30. X. Huang, S. Neretina, M.A. El-Sayed, Advanced Materials 21, 4880-4910 (2009)

31. TK. Sau, CJ. Murphy, J Am Chem Soc 126, 8648-8649 (2004)

32. C. Murphy, A. Gole, S. Hunyadi, C. Orendorff, Inorg Chem 45, 7544-7554 (2006)

33. M El-Sayed, Acc Chem Res 37, 326-333 (2004)

34. R. C. Price, R. L. Whetten, J. Am. Chem. Soc. 127, 13750 (2005)

35. S.K. Boruah, P.K. Boruah, P. Sarma, C. Medhi, O.K. Medhi, Advanced Materials Letters $3 ; 6$, 481-486 (2012)

36. O.V. Salata, Journal of Nanobiotechnology $2 ; 1$, 3 (2004) 
37. O. A. Sadik, F. Osonga, I. Yazgan, V. Kariuki, D. Luther, A. Jimenez, P. Le, RSC Adv. (2015)

38. S.S. Shankar, A. Ahmad, R. Pasricha, M. Sastry, Journal of Materials Chemistry $13 ; 7$, 1822-1826 (2003)

39. P. Elia, R. Zach, S. Hazan, S. Kolusheva, Z. Porat, Yehuda, International Journal of Nanomedicine 9, 4007-4021 (2014)

40. C. Subramaniam, T. Pradeep, J. Chakrabarti, Phys. Rev. Lett. 95, 164501 (2005)

41. P. K. Jain, K. S. Lee, I. H. El-Sayed, M. A. ElSayed, J. Phys.Chem. B 110, 7238 (2006)

42. G. Mie, Ann. Phys 25, 377 (1908)

43. P. K. Jain, X. Huang, I. H. El-Sayed, M. A. ElSayed, Acc.Chem. Res. 41, 1578 (2008)

44. S. Link, M. B. Mohamed, M. A. El-Sayed, J. Phys. Chem. B 103, 3073 (1999)

45. W. Rechberger, A. Hohenau, A. Leitner, J. R. Krenn, B. Lamprecht, F. R. Aussenegg, Opt. Commun. 220, 137 (2003)

46. S. Link, M. A. El-Sayed, J. Phys. Chem. B 103, 4212 (1999)

47. T. Itoh, T. Asahi, H. Masuhara, Appl. Phys. Lett. 79,1667 (2001)

48. K. H. Su, Q. H. Wei, X. Zhang, J. J. Mock, D. R. Smith, S. Schultz, Nano Lett. 3, 1087 (2003)

49. S. Srivastava, B. L. Frankamp, V. M. Rotello, Chem. Mater. 17, 487 (2005)

50. N. G. Khlebtsov, Anal. Chem. 80, 6620 (2008)

51. X. O. Liu, M. Atwater, J. H. Wang, Q. Huo, Colloids Surf. B 58, 3 (2007)

52. K. J. Fahnestock, M. Manesse, H. A. McIlwee, C. L. Schauer, R. Boukherroub, S. Szunerits, Analyst 134, 881 (2009)

53. J. J. Deng, Y. Song, Y. A. Wang, J. W. Di, Biosens. Bioelectron. 26, 615 (2010)

54. P. Englebienne, A. Van Hoonacker, M. Verhas, Analyst 126, 1645 (2001)

55. A. Prabhakar, S. Mukherji, Lab Chip 10, 3422 (2010)

56. J. Wang, Small 1, 1036 (2005)

57. J. Matsui, K. Akamatsu, N. Hara, D. Miyoshi, H. Nawafune, K. Tamaki, N. Sugimoto, Anal. Chem. 77, 4282 (2005)

58. F. Frederix, J. M. Friedt, K. H. Choi, W. Laureyn, A. Campitelli, D. Mondelaers, G. Maes, G. Borghs, Anal. Chem. 75, 6894 (2003)

59. R. A. Reynolds, C. A. Mirkin, R. L. Letsinger, J. Am. Chem. Soc. 122, 3795 (2000)

60. M. Frasconi, C. Tortolini, F. Botre, F. Mazzei, Anal. Chem. 82, 7335 (2010)

61. D. C. Hone, A. H. Haines, D. A. Russell, Langmuir 19,7141 (2003)

62. S. Watanabe, K. Yoshida, K. Shinkawa, D. Kumagawa, H. Seguchi, Colloids Surf. B 81, 570 (2010) 